\title{
New polyelectrolyte complex particles as colloidal dispersions based on weak synthetic and/or natural polyelectrolytes
}

\author{
M. Mihai ${ }^{*}$ C. A. Ghiorghiţă, I. Stoica, L. Niţă, I. Popescu, Ghe. Fundueanu \\ 'Petru Poni' Institute of Macromolecular Chemistry, 41A Grigore Ghica Voda Alley, 700487 Iasi, Romania
}

Received 13 October 2010; accepted in revised form 16 December 2010

\begin{abstract}
This study aims to evidence the formation of stable polyelectrolyte complex particles as colloidal dispersions using some weak polyelectrolytes: chitosan and poly(allylamine hydrochloride) as polycations and poly(acrylic acid) (PAA) and poly(2-acrylamido-2-methylpropanesulfonic acid - co - acrylic acid) (PAMPSAA) as polyanions. Polyelectrolyte complex particles as colloidal dispersion were prepared by controlled mixing of the oppositely charged polymers, with a constant addition rate. The influences of the polyelectrolytes structure and the molar ratio between ionic charges on the morphology, size, and colloidal stability of the complex particles have been deeply investigated by turbidimetry, dynamic light scattering and atomic force microscopy. A strong influence of polyanion structure on the values of molar ratio $n^{-} / n^{+}$when neutral complex particles were obtained has been noticed, which shifts from the theoretical value of 1.0 , observed when PAA was used, to 0.7 for PAMPSAA based complexes. The polyions chain characteristics influenced the size and shape of the complexes, larger particles being obtained when chitosan was used, for the same polyanion, and when PAMPSAA was used, for the same polycation.
\end{abstract}

Keywords: nanomaterials, polyelectrolyte complex, weak polyelectrolyte, dynamic light scattering, atomic force microscopy

\section{Introduction}

Oppositely charged polymers form polyelectrolyte complexes (PEC) after mixing of their solutions [1]. PECs are of interest due to their facile preparation and responsiveness to environmental stimuli. Moreover, using water as a solvent, PECs are attractive for biomedical applications. Examples include controlled drug delivery systems, enzyme and DNA carriers, surface modification of medical implants, membranes for cell culture and growth, biosensors, and nanostructured materials [2-5].

At relatively low concentrations and when one of the components is taken in excess, PEC formation can lead to stable colloidal dispersions [6-12]. The characteristics of the polyelectrolyte components (molecular weight, nature of ionic groups, charge density, and architecture) and the solvent (ionic strength, $\mathrm{pH}$ ) determine the internal structure of the particles. The level of aggregation is dominantly controlled by the concentration of the polymer solution. PECs as colloidal dispersions are interesting for industrial applications as coatings, auxiliary products in paper manufacturing, and for special purposes in biotechnology and medicine [13-15]. When weak polyelectrolytes are involved in PEC preparation, the molar ratio between charges when neutral complex particles were obtained is strongly influenced by the $\mathrm{pH}$ value of polyions solutions, conferring an amphoteric character to thePEC aggregates [16]. The amphoteric behavior of the PEC

\footnotetext{
${ }^{*}$ Corresponding author, e-mail: marcelas@icmpp.ro
}

(c) BME-PT 
particles, namely the sign of net surface charge, is determined by the positive or negative charges on the chain loops and chain ends on the particle surface.

Chitosan (CS) is a natural amino-polysaccharide (copolymer of $\beta$-(1-4) linked 2-acetamido-2-deoxyD-glycopyranose and 2-amino-2-deoxy-D-glycopyranose) produced by deacetylation of chitin from crustaceans' exoskeletons and fungal cell walls [17]. Sustainable interest in the biomedical application of chitosan to discourage bacterial adhesion and implant infection is stimulated strongly by its non-toxicity [18], biodegradability [19], and strong antibacterial properties [20]. It has been already shown that polyelectrolyte complexes and multilayers based on chitosan, by virtue of their biocompatibility, have valuable biomedical applications such as membranes for dialysis, packaging, coatings and wounds dressing, polyelectrolyte complex beads for controlled delivery of proteins, drugs, vaccines [21-28]. Promising results were obtained in application of chitosan based nonstoichiometric PECs as flocculants [29].

Our previous studies dealt with the preparation of PECs nanoparticles as stable colloidal dispersions using strong synthetic polyanions and synthetic or natural (CS) polycations. Also, the factors (polyions structure and molar mass, molar ratio between charges, addition order, titrant addition rate) which influence particles size, morphology, and storage stability were investigated [30-33]. This study aims to obtain PECs as colloidal dispersion using some weak polyelectrolytes: CS and poly(allylamine hidrochloride) $(\mathrm{PAH})$ as polycations and poly(acrylic acid) (PAA) and poly(2-acrylamido-2-methylpropanesulfonic acid - co - acrylic acid) (PAMPSAA) as polyanions. PAA and PAH, which have identical polymer backbones and differ only in the ionic side group, were chosen as model weak polymers for PEC formation. The influence of polyelectroytes structure and the molar ratio between ionic charges on the complex particles morphology, size, and colloidal stability have been deeply investigated by turbidimetry, dynamic light scattering (DLS) and atomic force microscopy (AFM).

\section{Materials and methods}

\subsection{Materials}

CS sample was kindly provided by Yaizu Suisankagaku Ind. (Shizuoka, Japan), as powder and was used without further purification. The viscometric average molar masses of CS sample, $M_{\mathrm{v}}=80000 \mathrm{~g} / \mathrm{mol}$, was estimated using Equation (1) [34]:

$[\eta]=6.95 \cdot 10^{-4} \cdot M_{\mathrm{v}}^{0.81}$

The intrinsic viscosity of CS solution in $0.3 \mathrm{M}$ $\mathrm{CH}_{3} \mathrm{COOH}-0.2 \mathrm{M} \mathrm{CH}_{3} \mathrm{COONa}(1: 1, \mathrm{v} / \mathrm{v})$ was measured with an Ubbelohde viscometer at $25 \pm 0.1{ }^{\circ} \mathrm{C}$.

The deacetylation degree was determined by two metods:

- by potentiometric titration, according to ref. [35], dissolving $\mathrm{CS}$ in a known excess of $\mathrm{HCl}$ and performing potentiometric titration with $0.1 \mathrm{~N}$ $\mathrm{NaOH}$, using an all-purpose titrator 716DMS Titrino, Metrohm (Herisau, Switzerland), equipped with a dosing unit and a combined glass electrode;

- from ${ }^{1} \mathrm{H}-\mathrm{NMR}$ spectra recorded on Bruker NMR model Avance DRX 400 (400 MHz) spectrometer (Bruker, Switzerland). The CS sample was dissolved in $\mathrm{D}_{2} \mathrm{O}$ and, to avoid the overlap between the signals of acetic acid and acetyl groups, some drops of $\mathrm{HCl}$ have been added to solubilize the polymer. The deacetylation degree was calculated as described before by Hirai et al. [36].

A deacetylation degree of about $82.5 \%$ was determined by both methods.

PAH, purchased from Aldrich (code no: 283215), with $M_{\mathrm{w}}=15000 \mathrm{~g} / \mathrm{mol}$, was used as received. PAA with a molar mass $M_{\mathrm{w}}=70000 \mathrm{~g} / \mathrm{mol}$ was synthesized and purified according to ref. [37]. The copolymer PAMPSAA, which contains $55 \mathrm{~mol} \%$ of 2-acrylamido-2-methylpropanesulfonic acid (AMPS) and $45 \mathrm{~mol} \%$ of acrylic acid (AA), was synthesized and purified according to ref. [38]. The intrinsic viscosity of the copolymer, determined in $1 \mathrm{M} \mathrm{NaCl}$ at $25^{\circ} \mathrm{C}$, was $[\eta]=0.72 \mathrm{dl} / \mathrm{g}$.

\subsection{Preparation of polyelectrolyte complexes as colloidal dispersions}

CS solution was prepared in $1 \mathrm{vol} \%$ acetic acid solution, and $0.02 \mathrm{M} \mathrm{NaCl}$. PAH and polyanions 
solutions, were prepared by dissolution of appropriate amounts in $0.02 \mathrm{M} \mathrm{NaCl}$ aqueous solution. The concentration of polycations solution was $5 \cdot 10^{-4} \mathrm{M}$ and that of polyanions solutions was $5 \cdot 10^{-3} \mathrm{M}$.

Dispersions of PECs were prepared at room temperature, by mixing the solutions of oppositely charged polyelectrolytes in appropriate proportions. The amount of polycation was kept constant within a complex series, while the amount of polyanion was varied according to the desired mixing molar ratio, $n^{-} / n^{+}$. The polyanion solution was added dropwise to the polycation solution, under magnetic stirring. A constant addition rate of $3.8 \mathrm{ml}$ polyanion/ $(\mathrm{ml}$ polycation $\cdot \mathrm{h})$ was used as optimum addition rate, taking into account the complex morphology and the preparation speed, according to our previous investigations on the formation of PECs as colloidal dispersion from synthetic polycations and polyanions [30-33]. After mixing, the formed dispersions were stirred $60 \mathrm{~min}$ and were characterized after $24 \mathrm{~h}$, if other conditions were not specified.

\subsection{Characterization methods}

Polyelectrolyte and potentiometric titrations were performed with the particle charge detector Mütek PCD 03 (BTG Instruments GmbH, Herrsching, Germany). The particle charge detector includes a Teflon piston, which moves up and down in a cylindrical Teflon cell containing the polyelectrolyte solution in the slit between the cylinder and piston. Displacement of the ion cloud around the polymer chains which are stuck on the porous wall of Teflon cylinder creates the streaming potential (measured in $\mathrm{mV}$ ) at the electrode pair located in upper and bottom parts of the cell. The measured streaming potential is linearly correlated with the charge density of polyelectrolytes and it becomes zero in case of charge neutrality. The concentration of the charged groups of each solution was evaluated by titration with a standard solution of a strong oppositely charged polyelectrolyte, poly(sodium ethylenesulfonate) or poly(diallyldimethyl-ammonium chloride), with a concentration of $10^{-3} \mathrm{M}$. The concentration of the charged groups in the examined solution was calculated from the amount of standard solution needed to reach the zero value of the streaming potential. All measurements were made at room temperature. Potentiometric titration was carried out between $\mathrm{pH} 2$ and 10, using $0.1 \mathrm{M}$ $\mathrm{NaOH}$ and $\mathrm{HCl}$, respectively.

The turbidity of the complex dispersions was characterized by the optical density at $\lambda=500 \mathrm{~nm}$ $\left(\mathrm{OD}_{500}\right)$, with a Lambda 900 spectrometer (PerkinElmer Co. Ltd., Massachusetts, USA), using deionized water to establish the baseline. At this wavelength, the used polyelectrolytes do not absorb. Optical density results were expressed as the average of at least three independent measurements.

DLS measurements of PEC dispersions were carried out using a Zetasizer 3000 (Malvern Instruments, Worcestershire, UK) equipped with a $10 \mathrm{~mW}$ $\mathrm{He}-\mathrm{Ne}$ laser $(633 \mathrm{~nm})$ as light source. The measurements were performed at a scattering angle of $90^{\circ}$, and the reported results are the average of two DLS independent measurements. The samples were kept at a constant temperature of $25^{\circ} \mathrm{C}$ during all experiments. DLS measures the light diffusion in particle dispersions, which can be interpreted using the Stokes-Einstein equation (Equation (2)) to yield the particle average hydrodynamic diameter, $D_{\mathrm{h}}$ :

$D_{\mathrm{h}}=\frac{k_{\mathrm{B}} T}{3 \pi \eta_{\mathrm{s}} D}$

where $k_{\mathrm{B}}$ is the Boltzmann constant, $T$ is the absolute temperature $(298 \mathrm{~K}), \eta_{\mathrm{s}}$ is the dynamic viscosity of the solvent (for water $0.8872 \mathrm{cP}$ ), and $D$ is the $z$ average translational diffusion coefficient. To get information about the size distribution of PEC particles, the size polydispersity was also included in the interpretation.

The shapes of PEC particles were examined by means of a SPM Solver PRO-M AFM (NT-MTD Co. Zelenograd, Moscow, Russia), using a high resolution 'Golden' silicon NSG10/Au/50 cantilever with an $\mathrm{Au}$ conductive coating. The topographic images were obtained in tapping mode and were repeated on different areas of the same sample. Prior to use, the silicon wafers substrates were carefully cleaned in two steps: first in 'piranha solution' followed by intensive rinsing with deionized water and second with the mixture $\mathrm{NH}_{4} \mathrm{OH} / \mathrm{H}_{2} \mathrm{O}_{2}$ /deionized water, at $70^{\circ} \mathrm{C}$, in ultrasonic bath, intensively rinsed with water and finally dried under a nitrogen flow. The clean silicon wafers substrates were immersed in PEC dispersions, identical with those used for DLS, for $20 \mathrm{~min}$, then three times each 
$1 \mathrm{~min}$ in distilled water and finally air-dried at room temperature (in a dust-free environment) for $48 \mathrm{~h}$.

\section{Results and discussion}

\subsection{Potentiometric titration of polyelectrolytes}

Although the value of streaming potential depends on numerous factors and can not be used as an absolute value (in the same way as $\zeta$-potential), the method of colloidal titration with streaming potential detection is useful to determine the point of zero charge $(p z c)$ in solutions of polyelectrolytes and colloidal dispersions. The $p z c$ was considered to be the numeric value of $\mathrm{pH}$ where the streaming current is zero $\mathrm{mV}$. When the $\mathrm{pH}$ is lower than the $p z c$ value, the system is below the $p z c$. Below the $p z c$, the acidic water donates more protons than hydroxide groups, and so the adsorbent surface is positively charged. Conversely, above $p z c$ the basic water donates more hydroxide groups than protons and the adsorbent surface is negatively charged. Figure 1 shows the potentiometric titrations curves for all polymers involved in this study, in the presence or absence of $\mathrm{NaCl}$.

As it is already known, the net charge on the weak polyelectrolyte molecules is affected by $\mathrm{pH}$ of their surrounding environment. The polyanions used in this study contain carboxyl groups. Below $p z c$, the carboxyl group can undergo protonation $\left(-\mathrm{COOH}_{2}{ }^{+}\right)$ at either the carbonyl oxygen or at the hydroxyl oxygen, and a small positive potential was obtained. Above $p z c$, located at $\mathrm{pH} 1.8$ for PAMPSAA and 2.2 for PAA (Figure 1), the presence of hydroxyl

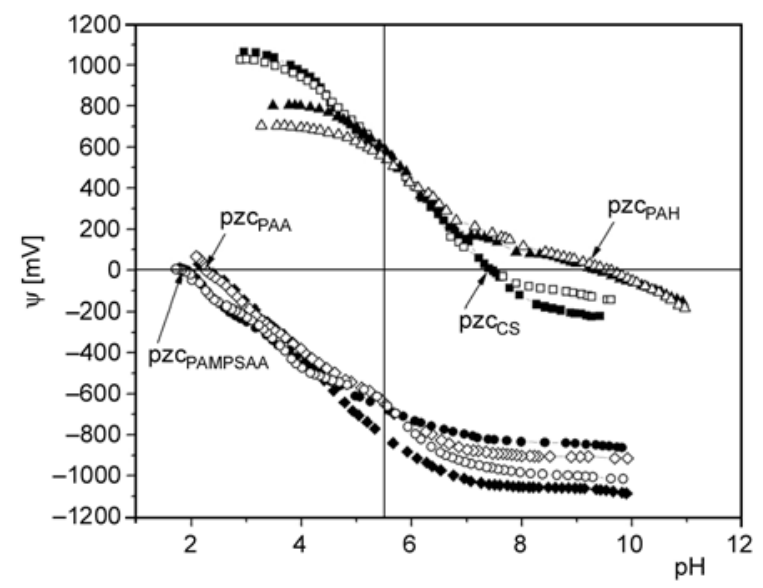

Figure 1. Potentiometric titration of CS (square), PAH (triangle), PAA (diamond), PAMPSAA (circles) in salt-free aqueous solution (close symbols), and $0.02 \mathrm{M} \mathrm{NaCl}$ aqueous solution (open symbols); $p z c=$ point of zero charges. ions induced the dissociation of carboxyl groups $\left(-\mathrm{COO}^{-}\right)$. Thus, above the $p z c$ the polyanions had negative streaming potential. At $p z c$ the ionization degree of polyanions is zero; e.g., the ionization degree of PAA at $\mathrm{pH}=2.2$ is 0.007 , taking into account that pKa of PAA is close to 4.3.

On the other hand, below $p z c$, in the presence of hydronium ions, the primary amino groups $\left(-\mathrm{NH}_{2}\right)$ of the polycations PAH and CS can be protonated $\left(-\mathrm{NH}_{3}{ }^{+}\right)$, leading to $p z c$ around $\mathrm{pH} 7.4$ for $\mathrm{CS}$ and 9.2 for PAH. Primary amines have protic hydrogens and therefore possess a small degree of acidity. Thus, above $p z c$ a proton from amine group can be dissociated $\left(-\mathrm{NH}^{-}\right)$, due to the strongly basic medium and a negative streaming potential is obtained.

In the presence of a small amount of low molecular weight salt $(0.02 \mathrm{M} \mathrm{NaCl})$, no significant changes in the shape of titration curves are observed, and the $p z c$ remains almost unchanged (Figure 1).

Taking into account the results obtained by potentiometric titration, for PECs preparation the $\mathrm{pH}$ value of polyions solutions was adjusted to 5.5 , to ensure a similar ionization degree of the complementary polyelectrolytes, suggested by the complementary streaming potential values at this $\mathrm{pH}$ : $\sim 600 \mathrm{mV}$ for polycations and $\sim 600 \mathrm{mV}$ for polyanions.

\subsection{Preparation and characterization of PECs}

\subsubsection{Turbidimetric titration}

The formation of colloidal PECs between all studied polyelectrolyte pairs was followed firstly by turbidimetric titration, $\mathrm{OD}_{500}$, as a function of the mixing ratio $n^{-} / n^{+}$, i.e., the molar ratio between anionic and cationic units (Figure 2).

It is well known that the turbidity values reflect the influence of some parameters that characterize the PECs dispersions such as concentration, size, shape and polydispersity $[6-8,14,30,31]$. Therefore, the increase of $\mathrm{OD}_{500}$ values with the increase of molar ratio between charges $n^{-} / n^{+}$, observed in Figure 2, could be assigned also to the increase of the sizes and polydispersities of the complex particles.

As it is already known, the values of molar ratio $n^{-} / n^{+}$corresponding to neutral PECs particles $\left(n^{-} / n^{+}\right)_{n}$ is strongly influenced by the structure of the complementary polyelectrolytes, namely by their structural ability to intrinsic compensate their ionic 


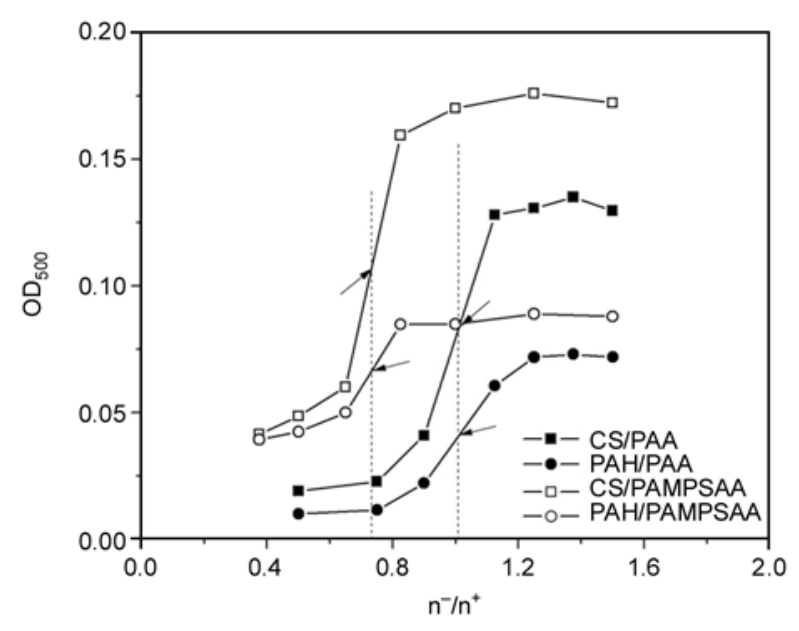

Figure 2. $\mathrm{OD}_{500}$ values as a function of the molar ratio between charges, $n^{-} / n^{+}$. The values are the mean of three independent experiments that deviated 0 $7 \%$. The continuous lines are 'hand-drawn' lines.

charges (polymer/polymer ion pairs) [39]. The values of molar ratio $\left(n^{-} / n^{+}\right)_{\mathrm{n}}$ was considered as the abscissa value of the rising curve corresponded to the one-half of the maximum of $\mathrm{OD}_{500}$. As Figure 2 shows, neutral complex particles were obtained at a molar ratio between charges of around $n^{-} / n^{+}=1$, when PAA was the polyanion, and around $n^{-} / n^{+}=$ 0.7 when PAMPSAA was used, irrespective the polycation structure. A deviation of the endpoint of polyelectrolyte interactions to lower values of $\left(n^{-} / n^{+}\right)_{\mathrm{n}}$ was also observed for other studied systems [31] and was ascribed to the difficulties in the intrinsic charge compensation between the complementary polyions.

For the same polyanion, higher values were obtained when CS was the polycation, along the whole range of molar ratio, $n^{-} / n^{+}$(Figure 2). These results may be ascribed to the lower flexibility of CS chain (which has a semi-rigid chain unlike the synthetic polycation which is flexible), which probably induced the formation of particles with higher size, reflected by higher $\mathrm{OD}_{500}$ values. For the same polycation, the $\mathrm{OD}_{500}$ values were higher when PAMPSAA was used, comparative with PAA.

\subsubsection{Dynamic light scattering}

DLS is a suitable method to monitor the formation of PEC particles as a function of the mixing molar ratio $n^{-} / n^{+}$. Figure 3 shows the DLS profiles on the PEC formation between CS and PAH, as starting polycations, and PAA and PAMPSAA as added polyanions.

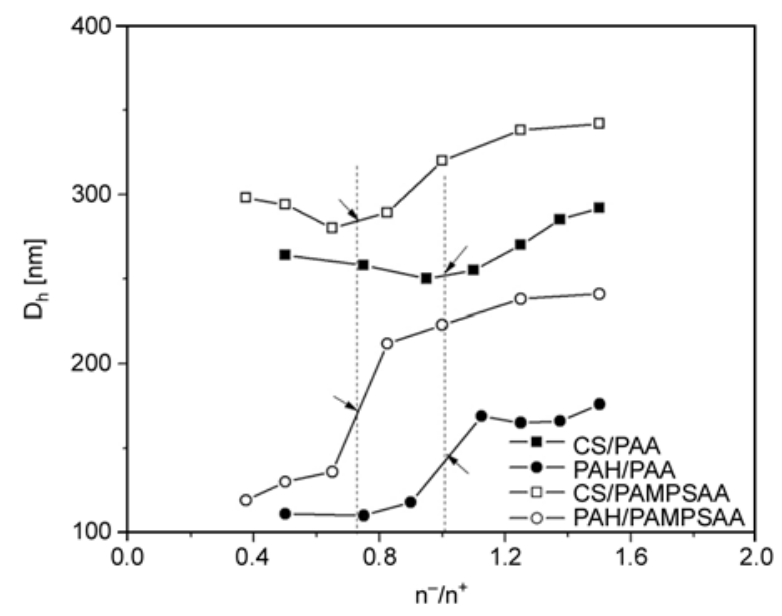

Figure 3. $D_{\mathrm{h}}$ of the PEC dispersions as a function of the molar ratio between charges $\left(n^{-} / n^{+}\right)$. The values are the mean of three independent experiments that deviated $0-7 \%$. The continuous lines are 'hand-drawn' lines.

The shape of the DLS curves was clearly influenced by the complementary polyelectrolytes structure. Thus, for PAH based complexes, $\mathrm{D}_{\mathrm{h}}$ values slowly increased up to about 0.9 and 0.6 when PAA and PAMPSAA was used, respectively, abruptly increased around $\left(n^{-} / n^{+}\right)_{\mathrm{n}}$, and remains almost constant after that. For CS based PECs, a slight decrease in the particle sizes was evident when CS was in excess, i.e. from 300 to $280 \mathrm{~nm}$ when PAMPSAA was used and from 265 to $248 \mathrm{~nm}$ when PAA was the added polyanion (Figure 3 ). This behavior suggests that below $\left(n^{-} / n^{+}\right)_{\mathrm{n}}$ the addition of polyanions allows both the formation of new particles, evidenced by the slow increase of the $\mathrm{OD}_{500}$ values (Figure 2), and also their rearrangement toward more compact structures. At values of molar ratio $n^{-} / n^{+}$higher than that corresponding to $\left(n^{-} / n^{+}\right)_{\mathrm{n}}$, the inter-particles aggregation took place, leading to the monotonous increase of the complex particles sizes. It seems that this kind of behavior is characteristic for CS based systems, as it was previously observed for PECs prepared with other polyanions $[29,33,40]$.

DLS results confirm the values of molar ratio $\left(n^{-} / n^{+}\right)_{\mathrm{n}}$, observed in Figures 2 , at $n^{-} / n^{+}=1$, when PAA was the polyanion, and around $n^{-} / n^{+}=0.7$ when PAMPSAA was used, irrespective the polycation structure. When PAMPSAA was used as polyanion, the particle sizes after the molar ratio $\left(n^{-} / n^{+}\right)_{\mathrm{n}}$ were higher than the sizes of the PEC formed with PAA as polyanion, irrespective of polycation nature 

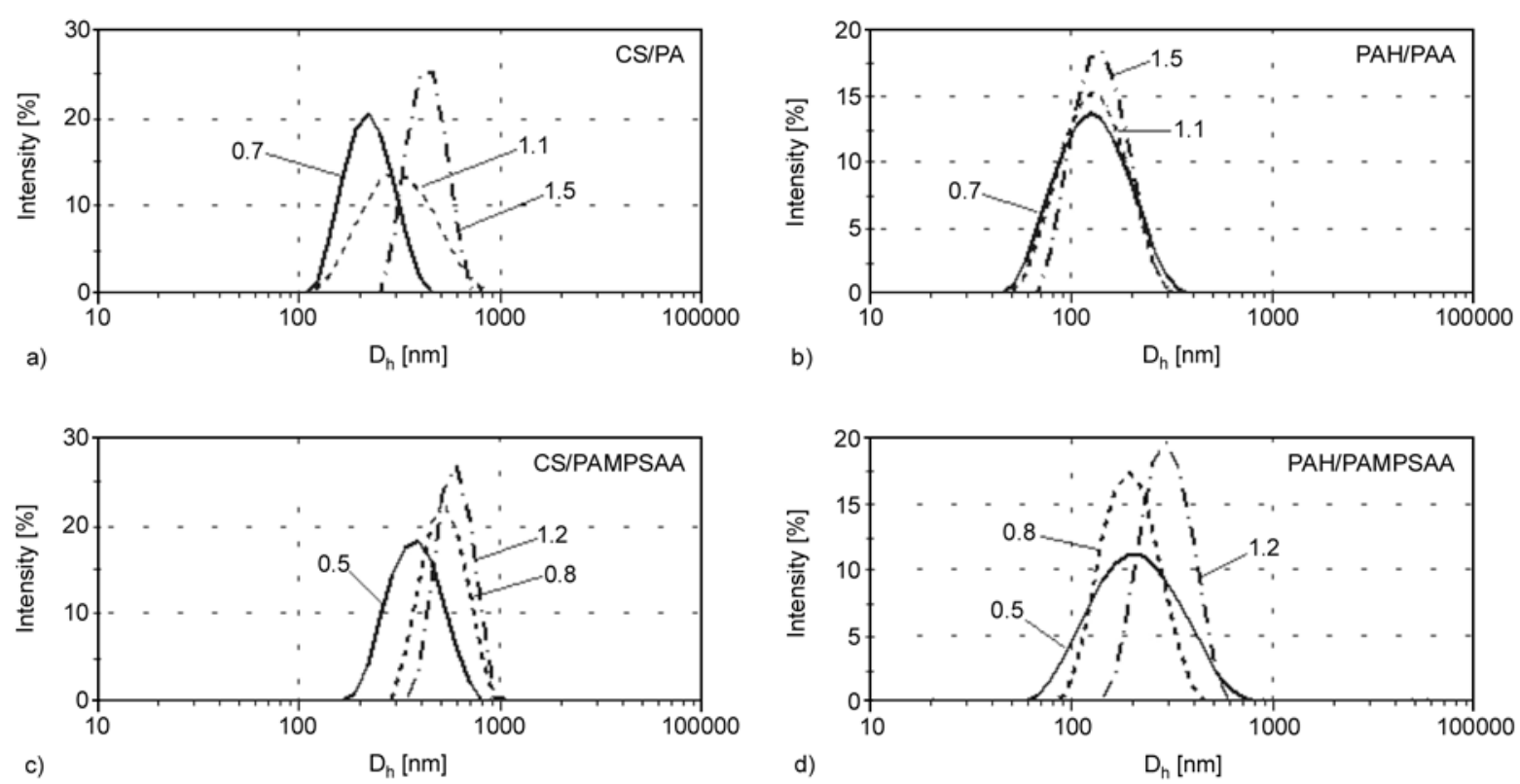

Figure 4. The particle size distribution analysis by intensity, obtain by DLS, for all studied systems and some selected molar ratios between charges

and molar ratio between charges. The copolymer contains 55\% AMPS units, with the ionic group attached by a longer spacer to the macromolecular backbone, with high flexibility, and a higher ability to compensate the ionic charges of the polycation. Thus, probably part of the AA and CS ionic sites are hindered, and remain extrinsic compensated by small ions, leading to values of molar ratio $\left(n^{-} / n^{+}\right)_{\mathrm{n}}$ lower than 1.0.

To evidence the influence of polyions structure on the size and polydispersity of PECs, the intensity of particle size distribution, determined by DLS, was also represented for all studied systems and some selected molar ratio between charges (Figure 4).

As Figure 4 shows, unimodal size distribution was observed for all studied systems, irrespective of the molar ratio between charges. At the same time, high values of polydispersity were observed at values of molar ratio $n^{-} / n^{+}$below and around $\left(n^{-} / n^{+}\right)_{\mathrm{n}}$, the highest values being obtained for the PAH/PAMPSAA pair. Moreover, a small decrease of the intensity of particle size distribution at $n^{-} / n^{+}=1.2$ was evident for all studied samples. This behavior suggests that the secondary aggregation by inter-particles interactions was not so fast and took place only when the polyanion was in excess, leading to a monotonous increase of the complex particles sizes (Figure 3).

\subsubsection{Atomic force microscopy}

In Figure 5, the AFM images of the PEC dispersion formed between all the studied polyions, at a molar ratio between charges of 1.2 are presented.

As Figure 5 shows, the adsorption of PEC particles on silicon wafers surface, resulted in an inhomogeneous mixture of smaller and bigger particles, for the studied samples. This is not surprising since the PEC particles showed a high polydispersity, evidenced by DLS measurements (Figure 4). Moreover, the AFM images confirm the results presented in Figure 3, namely bigger particle sizes can be observed for PECs prepared with CS as compared to PAH for the same polyanion (Figures $5 \mathrm{a}$ and $\mathrm{c}$ ), and with PAMPSAA as compared to PAA for the same polycation (Figures $5 \mathrm{~b}$ and $\mathrm{d}$ ).

The structure of polyanion strongly influenced the PECs particles shape: with PAA almost spherical particles were formed, but with PAMPSAA an irregular shape is evident, irrespective of polycation structure, probably due to the irregular compensation of the copolymer ionic sites.

The profiles of some selected particles of each sample (marked with a white line in Figures 5a-d) are presented in Figure 6.

Even if the interaction with the silica surface could induce conformational changes in the individual PEC particles, due to electrostatic interactions dur- 


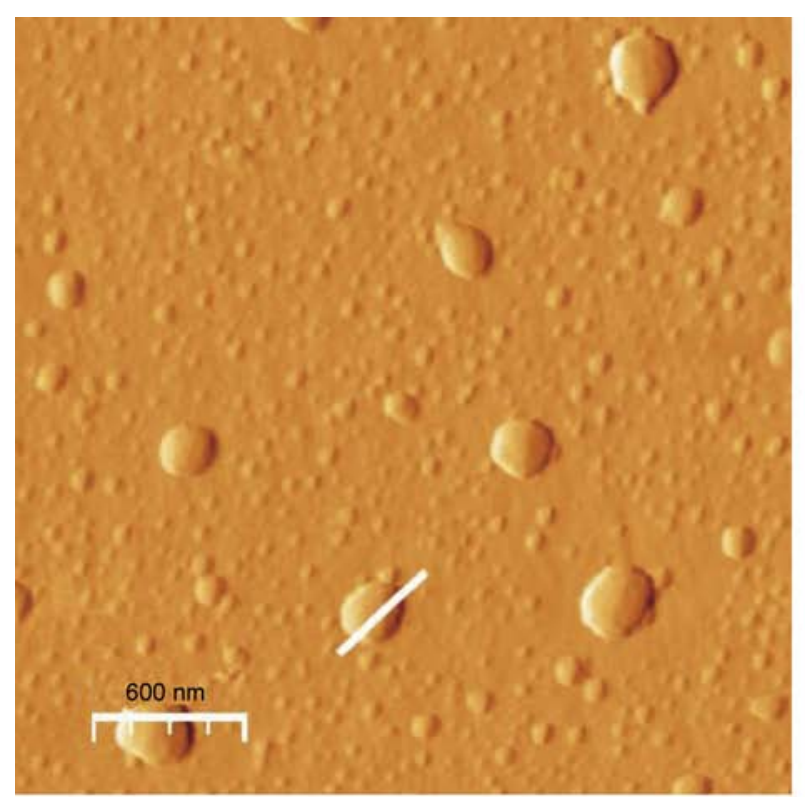

a) CS/PAA

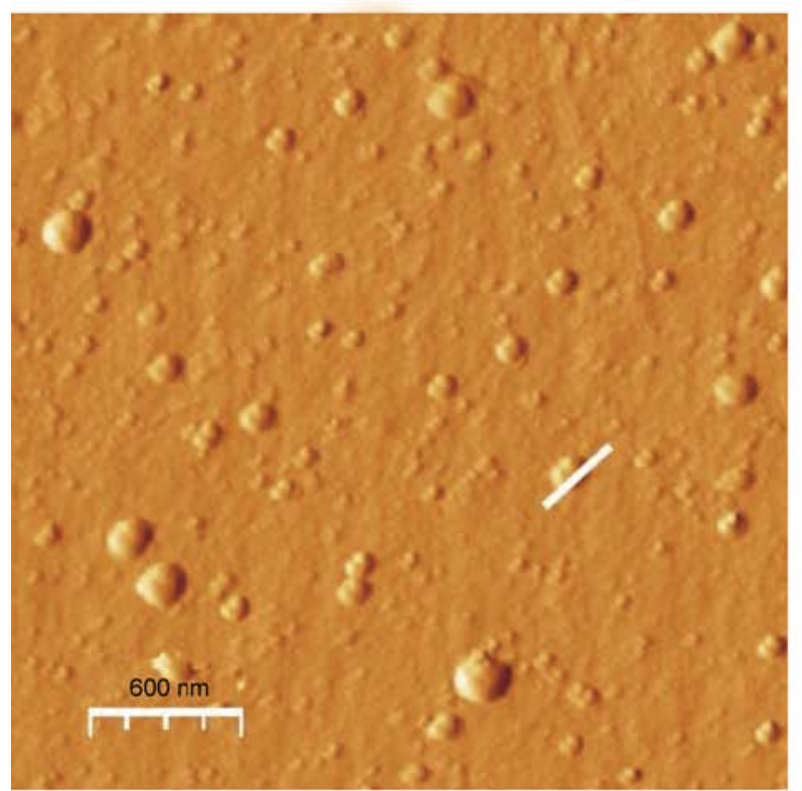

c) PAH/PAA

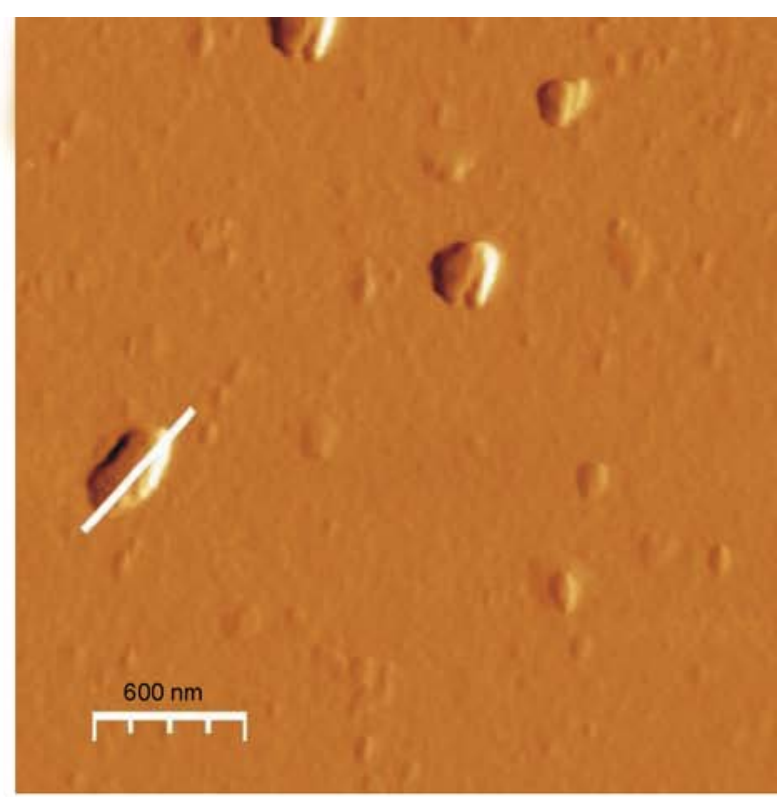

b) CS/PAMPSAA

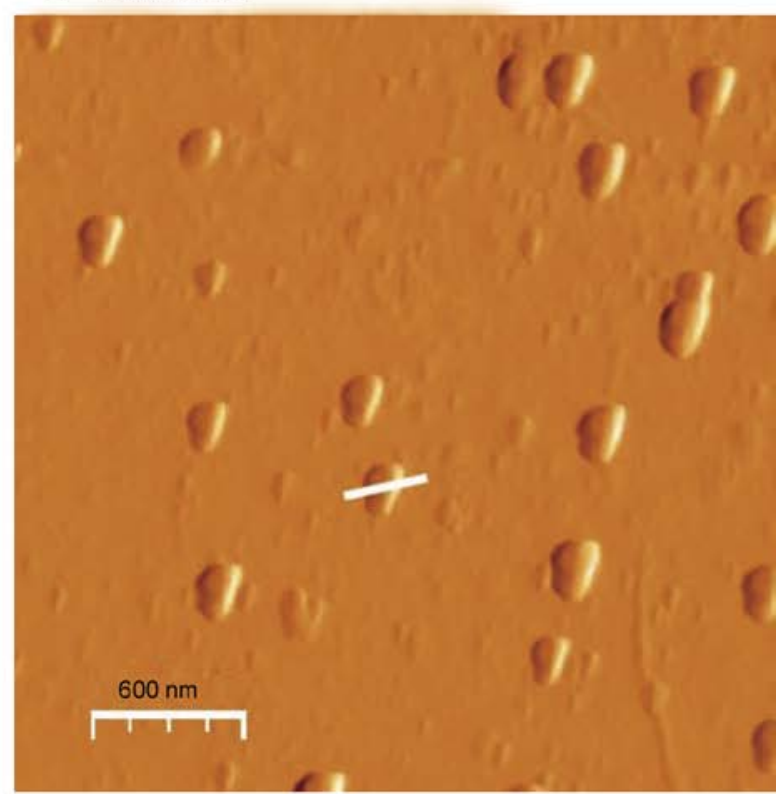

d) PAH/PAMPSAA

Figure 5. Tapping mode amplitude AFM images of the complex particles at a molar ratio between charges of 1.2, adsorbed on the silicon wafers

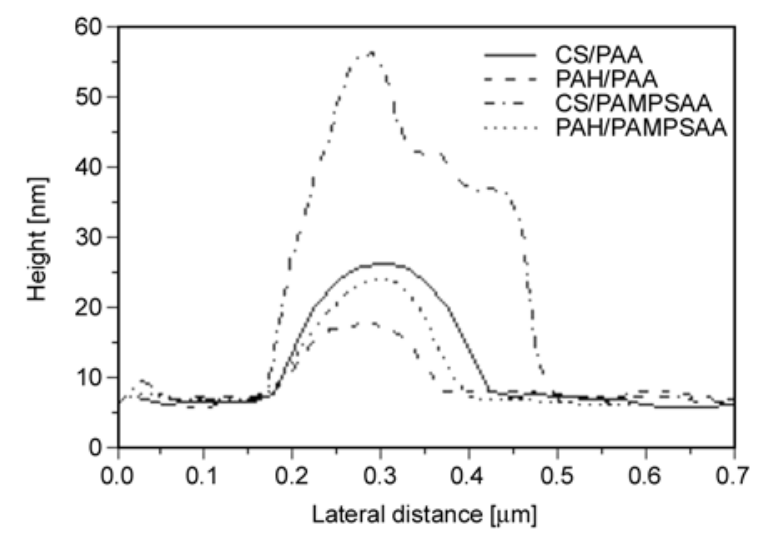

Figure 6. AFM profiles of some selected PEC particles (see white lines labeled particles in Figure 5) adsorbed on silicon wafers ing adsorption, after the air-drying procedure, the PECs particles still display 3D structures, the values of particles height increasing with their diameter, irrespective of the polyion pair involved in their preparation. Moreover, the values of the particles height were in agreement with the hydrodynamic diameter values (Figure 3), the smallest values being obtained for PAH/PAA complex particles and the highest for CS/PAMPSAA particles. 


\subsection{Colloidal stability}

When the complexation takes place between polyelectrolytes having significantly different molecular weights, weak ionic groups, in nonstoichiometric mixing ratios and under dilute conditions, the reaction mixture develops a colloidal, Tyndall effect. This behavior is ascribed to PECs structure, consisting of a neutral core surrounded by the polyelectrolyte in excess, which stabilizes PECs particles against aggregation, to provide practical nanoscale and microscale products $[2,6,9,11]$. The excess polyelectrolyte dictates the conditions for stability in different medium conditions. A very important characteristic of the PECs as colloidal dispersion is their colloidal stability. Storage colloidal stability of PECs is very sensitive to the structure of the complementary polyions, to the molar ratio between charges, i.e., the complex is closer or further to the molar ratio $\left(n^{-} / n^{+}\right)_{\mathrm{n}}$, to the polyions addition order, especially when the complementarities of oppositely charged polyions is disturbed by their structure, and very much to the titrant addition rate, as it was previously demonstrated for PECs prepared with strong polyanions [31-33]. Therefore, the particle sizes, $D_{\mathrm{h}}$, measured after $24 \mathrm{~h}$ from preparation were compared with those measured after two weeks of storage at room temperature $\left(\sim 22^{\circ} \mathrm{C}\right)$, without stirring (data not shown here). A high colloidal stability, i.e., low changes in the particle sizes, was found for PAH based PECs, along the whole range of investigated molar ratios between charges. As concerns the CS based PECs, when the polyanion is in excess the values of the particle sizes only slightly changed, the differences being more significant when CS was in excess, compared with those measured after $24 \mathrm{~h}$, the increase of the particles size being in the range of $15-30 \mathrm{~nm}$. These differences can be ascribed to the possible interactions between the primary aggregates, accompanied by rearrangements of chains and the formation of compact particles with higher sizes.

\section{Conclusions}

Two polyanions, PAA and a copolymer of AMPS with AA, and two weak polycations, $\mathrm{PAH}$ and $\mathrm{CS}$, were used in the preparation of some PEC dispersions. Turbidimetry, DLS and AFM have been used as complementary methods to describe the charac- teristics of the PEC dispersions as a function of the polyions structure and the ratio between charges.

The value of molar ratio $\left(n^{-} / n^{+}\right)_{\mathrm{n}}$ was clearly influenced by the polyanion structure, and was located at about 1.0 and 0.7 when PAA and PAMPSAA were respectively involved in the complexes preparation, irrespective of the polycation structure. The deviation of the endpoint of polyelectrolyte interactions to lower values of $\left(n^{-} / n^{+}\right)_{\mathrm{n}}$, in the case of PAMPSAA based PECs, can be ascribed to the presence of AMPS groups, which probably hindered the AA groups, conducting to a partially intrinsic compensate of carboxylic groups by the small counterions. The AFM images indicated that the adsorbed particles prepared with a molar ratio between charges of $n^{-} / n^{+}=1.2$, appeared as compact particles, their size and shape being strongly influenced by the complementary polyelectrolytes structure.

A high colloidal stability was found for PAH based PECs, on the whole range of investigated molar ratios between charges, and after the $\left(n^{-} / n^{+}\right)_{\mathrm{n}}$ for the CS based PECs.

\section{Acknowledgements}

The financial support of European Social Fund - 'Cristofor I. Simionescu' Postdoctoral Fellowship Program (ID POSDRU/89/1.5/S/55216) is gratefully acknowledged.

Abbreviations
\begin{tabular}{|l|l|}
\hline AFM & atomic force microscopy \\
\hline CS & chitosan \\
\hline$D_{\mathrm{h}}$ & hydrodynamic diameter \\
\hline DLS & dynamic light scattering \\
\hline$n^{-} / n^{+}$ & the molar ratio between anionic and cationic units \\
\hline$\left(n^{-} / n^{+}\right)_{\mathrm{n}}$ & $\begin{array}{l}\text { the molar ratio between anionic and cationic units } \\
\text { when neutral complex particles were obtained }\end{array}$ \\
\hline OD 500 & optical density at $\lambda=500$ nm \\
\hline PAA & poly(acrylic acid) \\
\hline PAH & poly(allylamine hidrochloride $)$ \\
\hline PAMPSAA & $\begin{array}{l}\text { poly(2-acrylamido-2-methylpropanesulfonic acid } \\
- \text { co- acrylic acid })\end{array}$ \\
\hline PI & polydispersity index \\
\hline PEC & polyelectrolyte complexes \\
\hline$p z c$ & point of zero charge \\
\hline
\end{tabular}

\section{References}

[1] Drăgan S., Cristea M.: Polyelectrolyte complexes. Formation, characterization and applications. in 'Recent research developments in polymer science' (ed.: Pandalai S. G.) Transworld Research Network, Trivandrum, Kerala, Vol 7, 149-181 (2003). 
[2] Tripathy S. K., Kumar J., Nalwa H. S.: Handbook of polyelectrolytes and their applications. Vol. 1-3, American Scientific Publishers, Los Angeles (2002).

[3] Thünemann A. F.: Polyelectrolyte-surfactant complexes (synthesis, structure and materials aspects). Progress in Polymer Science, 27, 1473-1572 (2002). DOI: 10.1016/S0079-6700(02)00017-5

[4] Liu T., Burger C., Chu B.: Nanofabrication in polymer matrices. Progress in Polymer Science, 28, 5-26 (2003).

DOI: 10.1016/S0079-6700(02)00077-1

[5] Shi X., Shen M., Mohwald H.: Polyelectrolyte multilayer nanoreactors toward the synthesis of diverse nanostructured materials. Progress in Polymer Science, 29, 987-1019 (2004).

DOI: $10.1016 /$ j.progpolymsci.2004.07.001

[6] Buchhammer H-M., Mende M., Oelmann M.: Preparation of monodisperse polyelectrolyte complex nanoparticles in dilute aqueous solution. Progress in Colloid and Polymer Science, 124, 98-102 (2003).

DOI: $10.1007 / \mathrm{b} 12146$

[7] Gärdlund L., Wågberg L., Gernandt R.: Polyelectrolyte complexes for surface modification of wood fibres: II. Influence of complexes on wet and dry strength of paper. Colloids and Surfaces A: Physicochemical and Engineering Aspects, 218, 137-149 (2003).

DOI: 10.1016/S0927-7757(02)00588-5

[8] Nyström R. G., Rosenholm J. B., Nurmi K.: Flocculation of semidilute calcite dispersions induced by anionic sodium polyacrylate-cationic starch complexes. Langmuir, 19, 3981-3986 (2003).

DOI: $10.1021 / 1 \mathrm{a} 034037 \mathrm{j}$

[9] Müller M., Kessler B., Richter S.: Preparation of monomodal polyelectrolyte complex nanoparticles of PDADMAC/poly(maleic acid-alt- $\alpha$-methylstyrene) by consecutive centrifugation. Langmuir, 21, 7044-7051 (2005).

DOI: $10.1021 / 1 \mathrm{a} 050716 \mathrm{~d}$

[10] Paneva D., Mespouille L., Manolova N., Degée P., Rashkov I., Dubois P.: Comprehensive study on the formation of polyelectrolyte complexes from (quaternized) poly[2-(dimethylamino)ethyl methacrylate] and poly(2-acrylamido-2-methylpropane sodium sulfonate). Journal of Polymer Science Part A: Polymer Chemistry, 44, 5468-5479 (2006).

DOI: $10.1002 /$ pola.21594

[11] Gummel J., Boué F., Demé B., Cousin F.: Charge stoichiometry inside polyelectrolyte-protein complexes: A direct SANS measurement for the PSSNa-lysozyme system. The Journal of Physical Chemistry B, 110, 24837-24846 (2006).

DOI: $10.1021 / j p 064383 \mathrm{k}$
[12] Hartig S. M., Carlesso G., Davidson J. M., Prokop A.: Development of improved nanoparticulate polyelectrolyte complex physicochemistry by nonstoichiometric mixing of polyions with similar molecular weights. Biomacromolecules, 8, 265-272 (2007).

DOI: $10.1021 / \mathrm{bm} 0604754$

[13] Petzold G., Nebel A., Buchhammer H-M., Lunkwitz K.: Preparation and characterization of different polyelectrolyte complexes and their application as flocculants. Colloid and Polymer Science, 276, 125-130 (1998).

DOI: $10.1007 / \mathrm{s} 003960050219$

[14] Reihs T., Müller M., Lunkwitz K.: Preparation and adsorption of refined polyelectrolyte complex nanoparticles. Journal of Colloid and Interface Science, 271, 69-79 (2004).

DOI: $10.1016 /$ j.jcis.2003.11.019

[15] Sui Z., Jaber J. A., Schlenoff J. B.: Polyelectrolyte complexes with $\mathrm{pH}$-tunable solubility. Macromolecules, 39, 8145-8152 (2006).

DOI: $10.1021 / \mathrm{ma} 061098 \mathrm{q}$

[16] Kötz J., Philipp B., Sigitov V., Kudaibergenov S., Bekturov E. A.: Amphoteric character of polyelectrolyte complex particles as revealed by isotachophoresis and viscometry. Colloid and Polymer Science, 266, 906912 (1988).

DOI: $10.1007 / \mathrm{BF} 01410845$

[17] Kubota N., Kikuchi Y.: Macromolecular complexes of chitosan. in 'Polysaccharides: Structural diversity and functional versatility' (ed.: Dumitriu S.) Marcel Dekker, New York, 595-628 (1998).

[18] Franklin J. J., Snow A.: Biochemistry of antimicrobial action. Chapman Hall, London (1981).

[19] Lee K. Y., Ha W. S., Park W. H.: Blood compatibility and biodegradability of partially $\mathrm{N}$-acylated chitosan derivatives. Biomaterials, 16, 1211-1216 (1995). DOI: $10.1016 / 0142-9612(95) 98126-Y$

[20] Rabea E. I., Badawy M. E-T., Stevens C. V., Smagghe G., Steurbaut W.: Chitosan as antimicrobial agent: Applications and mode of action. Biomacromolecules, 4, 1457-1465 (2003). DOI: $10.1021 / \mathrm{bm} 034130 \mathrm{~m}$

[21] Yan X-L., Khor E., Lim L-Y.: Chitosan-alginate films prepared with chitosans of different molecular weights. Journal of Biomedical Materials Research Part B: Applied Biomaterials, 58, 358-365 (2001). DOI: $10.1002 / \mathrm{jbm} .1029$

[22] Wang L., Khor E., Wee A., Lim L-Y.: Chitosan-alginate PEC membrane as a wound dressing: Assessment of incisional wound healing. Journal of Biomedical Materials Research, 63, 610-618 (2002).

DOI: $10.1002 / \mathrm{jbm} .10382$

[23] Rinaudo M.: Chitin and chitosan: Properties and applications. Progress in Polymer Science, 31, 603-632 (2006).

DOI: 10.1016/j.progpolymsci.2006.06.001 
[24] Shi X., Du Y., Sun L., Zhang B., Dou A.: Polyelectrolyte complex beads composed of water-soluble chitosan/alginate: Characterization and their protein release behavior. Journal of Applied Polymer Science, 100, 4614-4622 (2006).

DOI: 10.1002/app.23021

[25] Bratskaya S., Marinin D., Simon F., Synytska A., Zschoche S., Busscher H. J., Jager D., van der Mei H. C.: Adhesion and viability of two enterococcal strains on covalently grafted chitosan and chitosan/ $/$-carrageenan multilayers. Biomacromolecules, 8, 2960 2968 (2007).

DOI: $10.1021 / \mathrm{bm} 700620 \mathrm{j}$

[26] Kang H-S., Park S-H., Lee Y-G., Son T-I.: Polyelectrolyte complex hydrogel composed of chitosan and poly $(\gamma$-glutamic acid) for biological application: Preparation, physical properties, and cytocompatibility. Journal of Applied Polymer Science, 103, 386-394 (2007).

DOI: $10.1002 / a p p .24623$

[27] Crini G., Badot P-M.: Application of chitosan, a natural aminopolysaccharide, for dye removal from aqueous solutions by adsorption processes using batch studies: A review of recent literature. Progress in Polymer Science, 33, 399-447 (2008).

DOI: 10.1016/j.progpolymsci.2007.11.001

[28] Paneva D., Ignatova M., Manolova N., Rashkov I.: Novel chitosan-containing micro- and nanofibrous materials by electrospinning: Preparation and biomedical application. in 'Nanofibers: Fabrication, performance and applications' (ed.: Chang W. N.) Nova Science Publishers, New York, 73-151 (2009).

[29] Mihai M., Dragan E. S.: Chitosan based nonstoichiometric polyelectrolyte complexes as specialized flocculants. Colloids and Surfaces A: Physicochemical and Engineering Aspects, 346, 39-46 (2009).

DOI: $10.1016 /$ j.colsurfa.2009.05.021

[30] Dragan E. S., Schwarz S.: Polyelectrolyte complexes. VII. Complex nanoparticles based on poly(sodium 2acrylamido-2-methylpropanesulfonate) tailored by the titrant addition rate. Journal of Polymer Science Part A: Polymer Chemistry, 42, 5244-5252 (2004). DOI: $10.1002 /$ pola.20388

[31] Dragan E. S., Mihai M., Schwarz S.: Polyelectrolyte complex dispersions with a high colloidal stability controlled by the polyion structure and titrant addition rate. Colloids and Surfaces A: Physicochemical and Engineering Aspects, 290, 213-221 (2006).

DOI: $10.1016 /$ j.colsurfa.2006.05.022
[32] Mihai M., Dragan E. S., Schwarz S., Janke A.: Dependency of particle sizes and colloidal stability of polyelectrolyte complex dispersions on polyanion structure and preparation mode investigated by dynamic light scattering and atomic force microscopy. The Journal of Physical Chemistry B, 111, 8668-8675 (2007). DOI: $10.1021 / j p 071655 q$

[33] Dragan E. S., Mihai M., Schwarz S.: Complex nanoparticles based on chitosan and ionic/nonionic strong polyanions: Formation, stability, and application. ACS Applied Materials and Interfaces, 1, 12311240 (2009).

DOI: 10.1021/am900109u

[34] Gamzazade A. I., Šlimak V. M., Skljar A. M., Štykova E. V., Pavlova S. A., Rogožin S. V.: Investigation of the hydrodynamic properties of chitosan solutions. Acta Polymerica, 36, 420-424 (1985). DOI: $10.1002 /$ actp.1985.010360805

[35] Jiang X., Chen L., Zhong W.: A new linear potentiometric titration method for the determination of deacetylation degree of chitosan. Carbohydrate Polymers, 54, 457-463 (2003). DOI: $10.1016 /$ j.carbpol.2003.05.004

[36] Hirai A., Odani H., Nakajima A.: Determination of degree of deacetylation of chitosan by ${ }^{1} \mathrm{H}$ NMR spectroscopy. Polymer Bulletin, 26, 87-94 (1991).

DOI: $10.1007 / \mathrm{BF} 00299352$

[37] Dragan S., Cristea M., Luca C., Simionescu B. C.: Polyelectrolyte complexes. I. Synthesis and characterization of some insoluble polyanion-polycation complexes. Journal of Polymer Science Part A: Polymer Chemistry, 34, 3485-3494 (1996).

DOI: $10.1002 /($ SICI)1099-0518(199612)34:17<3485::

$$
\text { AID-POLA3>3.0.CO;2-U }
$$

[38] Stoilova O., Koseva N., Manolova N., Rashkov I.: Polyelectrolyte complex between chitosan and poly(2acryloylamido-2-methylpropanesulfonic acid). Polymer Bulletin, 43, 67-73 (1999). DOI: $10.1007 / \mathrm{s} 002890050534$

[39] Dubas S. T., Schlenoff J. B.: Polyelectrolyte multilayers containing a weak polyacid: Construction and deconstruction. Macromolecules, 34, 3736-3740 (2001). DOI: $10.1021 / \mathrm{ma} 001720 \mathrm{t}$

[40] Dinu I. A., Mihai M., Dragan E. S.: Comparative study on the formation and flocculation properties of polyelectrolyte complex dispersions based on synthetic and natural polycations. Chemical Engineering Journal, 160, 115-121 (2010). DOI: $\underline{10.1016 / \mathrm{j} . c e j .2010 .03 .018}$ 